\title{
MicroRNA Profile of Lung Tumor Tissues Is Associated with a High Risk Plasma miRNA Signature
}

\author{
Orazio Fortunato ${ }^{1}$, Carla Verri ${ }^{1}$, Ugo Pastorino ${ }^{2}$, Gabriella Sozzi ${ }^{1, *}$ and Mattia Boeri ${ }^{1}$ \\ 1 Unit of Tumor Genomics, Department of Experimental Oncology and Molecular Medicine, \\ Fondazione IRCCS Istituto Nazionale dei Tumori, Milan 20133, Italy; \\ orazio.fortunato@istitutotumori.mi.it (O.F.); carla.verri@istitutotumori.mi.it (C.V.); \\ mattia.boeri@istitutotumori.mi.it (M.B.) \\ 2 Unit of Thoracic Surgery, Fondazione IRCCS Istituto Nazionale dei Tumori, Milan 20133, Italy; \\ ugo.pastorino@istitutotumori.mi.it \\ * Correspondence: gabriella.sozzi@istitutotumori.mi.it; Tel.: +39-02-23-902-232; Fax: +39-02-23-902-928 \\ Academic Editor: Massimo Negrini \\ Received: 1 April 2016; Accepted: 28 June 2016; Published: 5 July 2016
}

\begin{abstract}
Lung cancer is the most common cause of cancer deaths worldwide. MicroRNAs (miRNAs) are short, non-coding RNAs that regulate gene expression. Many studies have reported that alterations in miRNA expression are involved in several human tumors. We have previously identified a circulating miRNA signature classifier (MSC) able to discriminate lung cancer with more aggressive features. In the present work, microarray miRNA profiling of tumor tissues collected from 19 lung cancer patients with an available MSC result were perform in order to find a possible association between miRNA expression and the MSC risk level. Eleven tissue mature miRNAs and six miRNA precursors were observed to be associated with the plasma MSC risk level of patients. Not one of these miRNAs was included in the MSC algorithm. A pathway enrichment analysis revealed a role of these miRNA in the main pathways determining lung cancer aggressiveness. Overall, these findings add to the knowledge that tissue and plasma miRNAs behave as excellent diagnostic and prognostic biomarkers, which may find rapid application in clinical settings.
\end{abstract}

Keywords: microRNA; microarray; liquid biopsy; lung cancer

\section{Introduction}

Lung cancer is the most frequent cause of cancer mortality worldwide, accounting for more than 1.4 million deaths per year [1]. Despite improvements in early diagnosis and new therapeutic strategies, the overall five-year survival remains only of $10 \%-20 \%$ [2]. Poor prognosis depends on several features: diagnosis at an advanced stage, tumor heterogeneity, and a limited understanding of lung cancer biology. Advances in genome-wide sequencing and microarray analysis are essential for a better definition of molecular mechanisms enhancing all types of cancer [3], as well as lung cancer [4].

MicroRNAs (miRNAs) are small, non-coding, 22 nt-long, RNAs able to bind complementary sequences of target mRNAs and to induce either their degradation or translational repression [5]. In mammals, miRNAs control the activity of more than $50 \%$ of all protein-coding genes [6]. miRNAs are expressed in a tissue specific manner, thereby greatly contributing to cell-type-specific profiles of protein expression. miRNAs potentially target hundreds of different mRNAs, thus regulating a wide variety of cellular processes [7]. To date, more than 1000 human miRNAs have been found in the genome, and a considerable number of them were found differentially-expressed in cancer cells compared to normal cells [8,9]. miRNAs can also be detected in biological fluids, such as plasma or serum, and can serve as circulating biomarkers $[10,11]$. They can be secreted via extracellular vesicles, 
such as exosomes [12], or by protein-miRNA complexes, such as high-density lipoprotein (HDL) or Argonaute2, protecting them from plasma RNase digestion [13]. So far, several miRNA-based liquid biopsy tools have been identified for cancer diagnosis and prognosis [14].

In previous studies we identified miRNA signatures with diagnostic and prognostic value in tumor, normal lung tissue, and plasma samples collected from patients enrolled in low-dose computed tomography (LDCT) screening trials $[15,16]$. In particular, we described a circulating miRNA signature classifier (MSC) able to identify lung cancer with more aggressive features. The MSC is composed by reciprocal ratios among 24 miRNAs, stratifying patients in three different groups: high, intermediate, and low, according to the risk of developing lethal lung cancer [17]. The 24 circulating miRNA were found to be involved in pathways regulating cellular aging, bronchi-alveolar and hematopoietic stem cells renewal, cell survival, and induction of angiogenesis [16]. In addition, among these, miR-660 was demonstrated to possess a therapeutic role in lung cancer cells and in vivo models [18].

In order to investigate if altered circulating miRNA levels associated with prognosis may reflect molecular changes at the level of the tumors, the miRNA expression profile of lung cancer tissues from 19 patients were compared according to the respective MSC risk level. A pathway enrichment analysis was further performed in order to define the biological processes regulated by identified miRNAs.

\section{Materials and Methods}

\subsection{Patients' Characteristics}

Plasma and tumor tissue samples were collected from 19 patients with a lung tumor detected during the LDCT screening trial performed at the Istituto Nazionale dei Tumori-Istituto Europeo di Oncologia (INT-IEO) [19]. Overall, between 2000 and 2005, a total of 1035 subjects were enrolled and $41(4 \%)$ developed lung cancer during the first five years of screening. The median age was 58 years (range 50-84), 739 (71\%) were men, average tobacco consumption was 26 cigarettes daily for 37 years (median pack/years $=40$ ), and $14 \%$ were former smokers. Both tissue and plasma specimens were collected from 19 patients at the time of surgery. All samples were obtained according to the Internal Review and the Ethics Boards of the Istituto Nazionale Tumori of Milan and all patients provided informed consent.

\subsection{MicroRNA Profiling in Plasma Samples}

Plasma samples were collected and RNA isolated as previously described $[16,20]$. Briefly, using mirVana PARIS Kit (Thermo Fisher Scientific, Waltham, MA, USA) total RNA was extracted from $200 \mu \mathrm{L}$ plasma samples and eluted in $50 \mu \mathrm{L}$ of buffer. MicroRNA expression was determined using the Megaplex Pools Protocol on microfluidic card type A (Thermo Fisher Scientific). For each microfluidic card (sample), the Ct of every miRNA was determined using the program SDS 2.2.2 (Thermo Fisher Scientific) and setting a threshold of 0.2 and a manual baseline from 3 to 18 cycles. The MSC algorithm was defined as previously described $[15,16,20]$. Briefly, since no housekeeping miRNAs were found in plasma samples, the MSC was composed by reciprocal ratios among 24 miRNAs differentially expressed between lung cancer patients and healthy individuals. The miRNAs composing the MSC are: miR-101-3p, miR-106a-5p, miR-126-5p, miR-133a, miR-140-3p, miR-140-5p, miR-142-3p, miR-145-5p, miR-148a-3p, miR-15b-5p, miR-16-5p, miR-17-5p, miR-197-3p, miR-19b-3p, miR-21-5p, miR-221-3p, miR-28-3p, miR-30b-5p, miR-30c-5p, miR-320a, miR-451a, miR-486-5p, miR-660-5p, and miR-92a-3p.

\subsection{MicroRNA Profiling in Tumor Tissue Samples}

Total RNA was extracted from fresh-frozen tumor samples available in the biobank of our institution, as previously described [16]. MiRNA labeling and hybridization was performed using $5 \mu \mathrm{g}$ of total TRIzol (Thermo Fisher Scientific) extracted RNA. The miRNA microarray (Ohio State University Comprehensive Cancer Center, version 2.0, Columbus, OH, USA) used contained probes for 460 mature miRNAs and 167 miRNA precursors spotted in quadruplicate with annotated active sites 
selected for oligonucleotide design. Hybridization signals were detected with streptavidin-Alexa-647 conjugate, and scanned images (Perkin-Elmer ScanArray XL5K Scanner) were quantified using the GeneSpring software version 7.2 (Silicon Genetics, Redwood City, CA, USA).

\subsection{Statistical and Bioinformatics Analyses}

On the microarray chips, after background subtraction and data transformation (to convert any negative value to 0.01), the average value of the four spots was normalized using a per-chip 50th percentile method that normalizes each chip on its median (data available as Table S2). Class comparison analyses were performed using BRB ArrayTools 4.2.1 software [21]. MicroRNAs differentially expressed between two classes were considered significant at the nominal 0.01 level of the univariate test based on 10,000 random permutations. Survival curves were estimated using the Kaplan-Meier method and were compared by the log-rank test [22].

\subsection{Pathways Enrichment Analysis}

Pathway enrichment analysis was performed considering only mature miRNA differentially expressed using both DIANA-mirPath V.2 and miRWalk2.0 online softwares [23,24]. For mirPath analysis, gene targets were predicted by microT-CDS and the results were merged using the pathway union mode with false discovery rate (FDR) correction, which calculate the merged $p$-value of all miRNAs for each pathway by applying Fisher's meta-analysis method. Pathways were considered significant with $p$-value $<0.01$ and microT threshold $=0.8$. For miRWalk analysis, predicted targets identified by miRWalk or Targetscan, and Kyoto Encyclopedia of Genes and Genomes (KEGG) pathways were considered significant having adjusted $p$-values $<0.05$ after FDR correction.

\section{Results}

\subsection{Patients' Characteristics and Prognostic Value of the miRNA Signature Classifier (MSC)}

Tumor specimens were collected at the time of surgery from 19 lung cancer patients enrolled in the INT-IEO LDCT-screening trial performed in our institute. Patients were all heavy smokers with a pack-years (PY) index higher than 20 and older than 50 years. The majority of tumors were adenocarcinoma (ADC, 73.7\%), while $15.8 \%$ squamous cell carcinoma (SCC) and $10.5 \%$ other tumors. As expected in a screening-detected tumors population, stage I were more frequent $(63.2 \%)$ than the higher stages $(36.8 \%)$, and the $57.9 \%$ of patients was still alive after five years of follow-up. Only one patient was censored after 3.1 years. From the same subjects, plasma samples were also available to perform the MSC test: $12(63.2 \%)$ patients resulted high, six (31.6\%) intermediate, and one (5.3\%) low risk MSC (Table 1). The five-year overall survival (OS) for the 12 patients with high MSC risk level was 31.3\%, while for the seven patients with intermediate or low MSC risk levels was $100 \%(p$-value $=0.007$, Figure 1$)$.

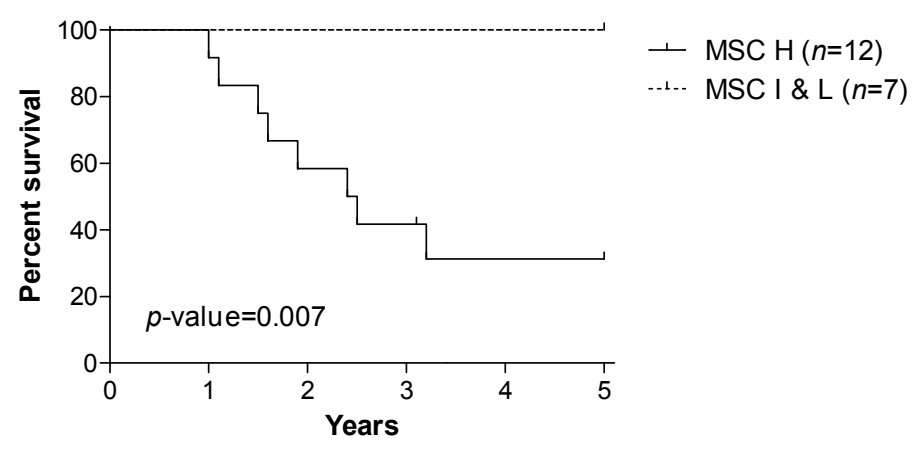

Figure 1. Kaplan Meier curves for 19 lung cancer patients in strata of the miRNA signature classifier (MSC) level of risk: high (H) vs. Iintermediate and low (I and L). $p$ for log rank test. 
Table 1. Clinical-pathological characteristics of 19 low-dose computed tomography (LDCT)-detected lung cancer cases of the Istituto Nazionale dei Tumori-Istituto Europeo di Oncologia (INT-IEO) screening trial with available plasma samples for the miRNA signature classifier (MSC) test.

\begin{tabular}{ccc}
\hline Clinico-Pathological Charachteristics & \multicolumn{3}{c}{ Trial INT-IEO $N=\mathbf{1 9}$} \\
\hline Gender & 12 & $(63.2 \%)$ \\
Male & 7 & $(36.8 \%)$ \\
Female & $57.5 \pm 5.6$ (s.d.) \\
\hline Age (years) & $60.3 \pm 23.8$ (s.d.) \\
\hline Smoking habit (Pack-Years index) & & \\
Histotype & 14 & $(73.7 \%)$ \\
ADC (adenocarcinoma) & 3 & $(15.8 \%)$ \\
SCC (squamous carcinoma) & 2 & $(10.5 \%)$ \\
\hline other & 12 & $(63.2 \%)$ \\
Stage & 7 & $(36.8 \%)$ \\
Ia-lb & & \\
II-III-IV & 11 & $(57.9 \%)$ \\
Status at 5 years & 8 & $(42.1 \%)$ \\
Alive & 12 & $(63.2 \%)$ \\
Dead & 7 & $(36.8 \%)$ \\
\hline MSC risk level &
\end{tabular}

\subsection{MicroRNA Expression in Tumor Tissue Associated with MSC Risk Level}

MicroRNA profiles of the 19 lung tumor tissues were analyzed using the microarray x-platform (Ohio State University Comprehensive Cancer Centre, version 2.0). By stratifying patients in MSC high versus intermediate and low risk level, class comparison analyses showed that the expression of 11 mature miRNAs and six miRNA precursors were significantly different at the nominal 0.01 level of the univariate test (Table 2). In particular, hsa-mir-520e, hsa-mir-518c-5p, and all six miRNA precursors were, resultingly, downmodulated in the high risk group, while hsa-mir-329-3p, hsa-mir-302d-3p, hsa-mir-520f, hsa-mir-511-5p, hsa-mir-509-3p, hsa-mir-519a-3p, hsa-mir-521, hsa-mir-520h, and hsa-mir-499a-5p were overexpressed.

Table 2. Class comparison analysis of microRNA expression in tumor tissue of lung cancer patients stratified according to MSC risk level: high (H) vs. Intermediate (I) and low (L).

\begin{tabular}{|c|c|c|c|c|c|}
\hline MicroRNA & $\begin{array}{c}\text { Parametric } \\
p \text {-Value }\end{array}$ & $\begin{array}{c}\text { Permutation } \\
p \text {-Value }\end{array}$ & $\begin{array}{c}\text { Geom Mean of MSC } \\
\text { H Patients }\end{array}$ & $\begin{array}{l}\text { Geom Mean of MSC } \\
\text { I \& L Patients }\end{array}$ & Fold-Change \\
\hline hsa-mir-210-prec & 0.0003 & $9.00 \times 10^{-4}$ & 782.18 & 1474.27 & 0.53 \\
\hline hsa-mir-520e & 0.0003 & $8.00 \times 10^{-4}$ & 382.79 & 528.74 & 0.72 \\
\hline hsa-mir-520h & 0.0006 & $4.00 \times 10^{-4}$ & 712.74 & 485.72 & 1.47 \\
\hline hsa-mir-7-2-prec & 0.0009 & 0.0011 & 762.86 & 1107.25 & 0.69 \\
\hline hsa-mir-329-3p & 0.0017 & 0.0016 & 299.35 & 277.83 & 1.08 \\
\hline hsa-mir-520f-3p & 0.002 & 0.0016 & 304.94 & 274.83 & 1.11 \\
\hline hsa-mir-511-5p & 0.002 & $8.00 \times 10^{-4}$ & 339.84 & 306.75 & 1.11 \\
\hline hsa-mir-521 & 0.0021 & $5.00 \times 10^{-4}$ & 746.92 & 525.49 & 1.42 \\
\hline hsa-mir-15a-prec & 0.0032 & 0.0031 & 390.85 & 489.14 & 0.8 \\
\hline hsa-mir-518c-5p & 0.004 & 0.0055 & 353.1 & 418.94 & 0.84 \\
\hline hsa-mir-147-prec & 0.005 & 0.0049 & 401.67 & 457.29 & 0.88 \\
\hline hsa-mir-302d-3p & 0.0054 & 0.0038 & 299.31 & 274.01 & 1.09 \\
\hline hsa-mir-499a-5p & 0.0058 & 0.0049 & 1726.93 & 1028.1 & 1.68 \\
\hline hsa-mir-125a-prec & 0.0062 & 0.0055 & 364.76 & 435.28 & 0.84 \\
\hline hsa-mir-138-2-prec & 0.0067 & 0.008 & 893.27 & 1298.2 & 0.69 \\
\hline hsa-mir-519a-3p & 0.0068 & 0.0036 & 2117.4 & 1501.89 & 1.41 \\
\hline hsa-mir-509-3p & 0.0099 & 0.0065 & 1204.47 & 908.23 & 1.33 \\
\hline
\end{tabular}

Type of univariate test used: two-sample $t$-test; permutation $p$-values for significant miRNAs were computed based on 10,000 random permutations; nominal significance level of each univariate test. 
Interestingly, by stratifying patients according to survival status at five years, only two mature miRNAs (hsa-mir-499a-5p and hsa-mir-429) and one miRNA precursor (hsa-mir-212-prec) were found differentially expressed (Supplementary Table S1), being only mir-499a-5p in common with the MSC related signature.

\subsection{Pathway Enrichment Analysis}

A pathway enrichment analysis was performed using the MirPath software and the 11 mature miRNAs. As reported in Table 3, important pathways contributing to tumor aggressiveness were regulated by at least four of these miRNAs. Among these, the following pathways were observed: proliferation pathways, such as $M A P K$ signaling pathway (regulated by six miRNAs) and the ErbB signaling pathway (by four miRNAs); pathways associated with epithelial to mesenchymal transition (EMT), such as the regulation of the actin cytoskeleton (by six miRNAs) and the TGF- $\beta$ signaling pathway (by four miRNAs) involved in cancer cell dissemination and metastasis formation; the cell survival PI3K-Akt signaling pathway (by five miRNAs) and in general pathways associated with non-small cell lung cancer (NSCLC, by four miRNAs), including the epithelial growth factor receptor (EGFR) signaling.

Table 3. List of Kyoto Encyclopedia of Genes and Genomes (KEGG) pathways regulated by at least four mature miRNAs differentially expressed between lung cancer patients with high and intermediate/low risk MSC. DIANA-mirPath V.2.

\begin{tabular}{cccc}
\hline KEGG Pathway & $p$-Value & \#Genes & \#miRNAs \\
\hline MAPK signaling pathway & $3.66 \times 10^{-15}$ & 51 & 6 \\
Regulation of actin cytoskeleton & $8.99 \times 10^{-9}$ & 40 & 6 \\
Hepatitis B & $1.68 \times 10^{-14}$ & 35 & 5 \\
Axon guidance & $3.39 \times 10^{-14}$ & 22 & 5 \\
Chronic myeloid leukemia & $5.98 \times 10^{-13}$ & 24 & 5 \\
PI3K-Akt signaling pathway & $1.49 \times 10^{-12}$ & 67 & 5 \\
Pancreatic cancer & $2.24 \times 10^{-11}$ & 23 & 5 \\
Transcriptional misregulation in cancer & $5.40 \times 10^{-9}$ & 34 & 5 \\
ErbB signaling pathway & $1.35 \times 10^{-11}$ & 25 & 4 \\
Neurotrophin signaling pathway & $2.21 \times 10^{-11}$ & 23 & 4 \\
TGF- $\beta$ signaling pathway & $1.34 \times 10^{-9}$ & 17 & 4 \\
Non-small cell lung cancer & $3.53 \times 10^{-8}$ & 15 & 4 \\
\hline
\end{tabular}

Way to merge results: pathway union with FDR correction; $p$-value threshold: 0.01; MicroT threshold: 0.8 .

In particular, hsa-mir-302d-3p, hsa-mir-329-3p, hsa-mir-519a-3p, and hsa-mir-520e-f were associated with multiple pathways. On the other hand, other miRNAs were associated with a just a few pathways specific to cancer development: hsa-mir-499a-5p, associated with proliferation signaling, and hsa-mir-511-5p and hsa-mir-520h with EMT (Figure 2). Similar results were also obtained using the miRWalk2.0 prediction software, where pathways in cancer, $M A P K$ signaling pathway, non-small cell lung cancer, Wnt signaling pathway, and the $E r b B$ signaling pathway were significantly $(p<0.05)$ regulated by at least four of these 11 mature miRNAs (Supplementary Figure S1). 

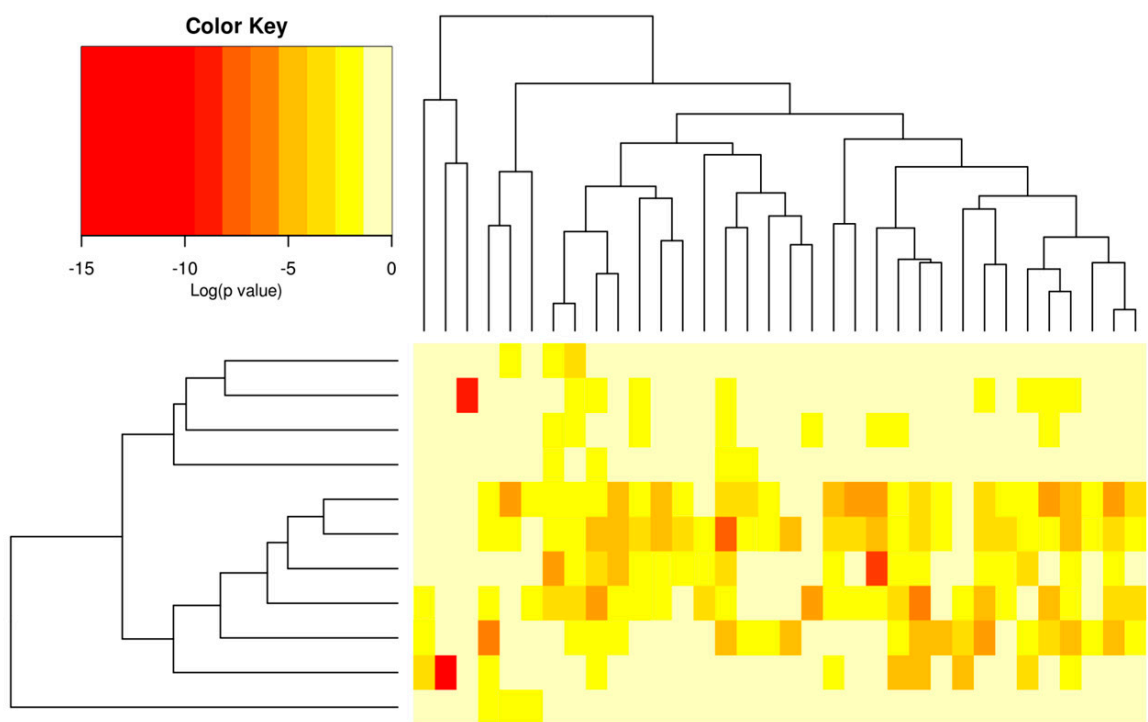

hsa-miR-518c-5p/microT-CDS nsa-miR-499a-5p/microT-CDS nsa-miR-511/microT-CDS hsa-miR-509-3p/microT-CDS hsa-miR-520f/microT-CDS hsa-miR-520e/microT-CDS hsa-miR-302d-3p/microT-CDS hsa-miR-519a-3p/microT-CDS hsa-miR-329/microT-CDS hsa-miR-520h/microT-CDS hsa-miR-521/microT-CDS

Way to merge results: pathway union with FDR correction

P-value threshold: 0.01

MicroT threshold: 0.8

Figure 2. Heat map of Kyoto Encyclopedia of Genes and Genomes (KEGG) pathway enrichment analysis using DIANA-mirPath V.2 and considering 11 mature miRNAs differentially expressed between lung cancer patients with high and intermediate/Low risk miRNA signature classifier (MSC).

\section{Discussion}

Gaining insight into the molecular changes that underlie aggressive lung cancers is of critical clinical relevance. The purpose of this work was to investigate in a subset of CT-detected lung cancer patients the association between plasma miRNA risk profile and their miRNA expression profile in tumor tissue.

We previously demonstrated that a plasma miRNA signature classifier (MSC), stratifying individuals undergoing lung cancer screening into three risk levels (high, intermediate, and low), predicted diagnosis and the overall survival (OS). Moreover, a high-risk miRNA circulating profile was demonstrated to identify malignancy and aggressive disease [15,17].

Analysis of the tissue miRNA profile of lung cancer patients according to prognosis showed differences in only three miRNAs, suggesting high heterogeneity of tumor samples within the two groups. On the other hand, when stratification was performed on the basis of their plasma miRNA risk level, a panel of 11 mature miRNAs and six differentially-expressed miRNA precursors were observed. The different results obtained by miRNA profiles suggest the clinical relevance of molecular stratification in identifying more homogenous subgroup of patients, for a more accurate patient management.

Interestingly, none of the 17 cancerous tissue miRNAs belong to the 24 plasma miRNA panel composing the MSC, supporting the hypothesis that circulating miRNAs are mostly contributed from tumor-surrounding changes, strongly affected by smoking carcinogen exposure, permissive for the developing of an aggressive disease. Accordingly, time dependency analysis showed that the MSC risk level was already positive up to two years before disease detection by LDCT [15]. Moreover, even 
after cancer removal, relapsing patients retained an elevated MSC level, reflecting the persistence of a host/microenvironment-related risk profile [17].

Published studies showed that mir-499a-5p is a tumor suppressor miRNA by targeting the VAV3 gene and its reduction correlate with poor clinical outcome in NSCLC. Experimental data show that mir-499a-5p overexpression induces apoptosis, and inhibits cell proliferation in vitro and NSCLC metastasis in vivo [25]. Mir-329, by targeting oncogenic MET, reduces cell proliferation, migration, invasion, and promotes apoptosis in lung cancer cell lines [26]. Mir-520e is involved in the NIK/p-ERK1/2/NF- $\kappa B$ signaling in hepatocarcinogenesis having as direct target NIK gene [27], while mir-519a acts in STAT3 pathways and is correlated with poor outcome in glioblastoma [28]. Finally, mir-302d-3p is a member of the mir-302/367 cluster that has a role in the regulation of cell signaling pathways involved in the cell cycle and inducing pluripotent stem cells [29,30].

The analysis of pathway enrichment regulated by these miRNAs displayed involvement of relevant biological processes, such as proliferation, growth factor receptor signaling, signal transduction, cell survival, dissemination, and metastasis; features that are altered in the more common cancers, and particularly in lung cancer.

\section{Conclusions}

These results indicate that the biological trait of lung tumors with a high-risk plasma miRNA profile is enriched for features related to disease aggressiveness, supporting the established prognostic role of plasma MSC classification in lung cancer patients enrolled in screening trials. Overall, these findings add to the knowledge that tissue and plasma miRNAs behave as excellent diagnostic and prognostic biomarkers which may find rapid application in clinical settings.

Supplementary Materials: The supplementary materials are available online at http:/ /www.mdpi.com/20763905/5/3/18/s1.

Acknowledgments: Supported by Investigator Grants No. 15928 (to UP), 14318 (to GS), and 12162 (Special Program "Innovative Tools for Cancer Risk Assessment and early Diagnosis", 5x1000) from the Italian Association for Cancer Research, Grant No. RF-2010 from the Italian Ministry of Health. M. Boeri was supported by a Fondazione Umberto Veronesi Fellowship.

Author Contributions: Orazio Fortunato, Carla Verri, Ugo Pastorino, Gabriella Sozzi and Mattia Boeri.conceived and designed the experiments; Orazio Fortunato, Carla Verri and Mattia Boeri performed the experiments; Orazio Fortunato, Carla Verri and Mattia Boeri analyzed the data; Orazio Fortunato, Carla Verri, Gabriella Sozzi and Mattia Boeri wrote the paper.

Conflicts of Interest: Gabriella Sozzi, Mattia Boeri, and Ugo Pastorino are co-inventors for three patent applications regarding the miRNA signature disclosed in this article.

\section{References}

1. Torre, L.A.; Bray, F.; Siegel, R.L.; Ferlay, J.; Lortet-Tieulent, J.; Jemal, A. Global cancer statistics, 2012. CA Cancer J. Clin. 2015, 65, 87-108. [CrossRef] [PubMed]

2. Siegel, R.; Ma, J.; Zou, Z.; Jemal, A. Cancer statistics, 2014. CA Cancer J. Clin. 2014, 64, 9-29. [CrossRef] [PubMed]

3. Ramaswamy, S.; Tamayo, P.; Rifkin, R.; Mukherjee, S.; Yeang, C.H.; Angelo, M.; Ladd, C.; Reich, M.; Latulippe, E.; Mesirov, J.P.; et al. Multiclass cancer diagnosis using tumor gene expression signatures. Proc. Natl. Acad. Sci. USA 2001, 98, 15149-15154. [CrossRef] [PubMed]

4. Yanaihara, N.; Caplen, N.; Bowman, E.; Seike, M.; Kumamoto, K.; Yi, M.; Stephens, R.M.; Okamoto, A.; Yokota, J.; Tanaka, T.; et al. Unique microRNA molecular profiles in lung cancer diagnosis and prognosis. Cancer Cell 2006, 9, 189-198. [CrossRef] [PubMed]

5. Bartel, D.P. MicroRNAs: Genomics, biogenesis, mechanism, and function. Cell 2004, 116, 281-297. [CrossRef]

6. Rodriguez, A.; Griffiths-Jones, S.; Ashurst, J.L.; Bradley, A. Identification of mammalian microRNA host genes and transcription units. Genome Res. 2004, 14, 1902-1910. [CrossRef] [PubMed]

7. Krol, J.; Loedige, I.; Filipowicz, W. The widespread regulation of microRNA biogenesis, function and decay. Nat. Rev. Genet. 2010, 11, 597-610. [CrossRef] [PubMed] 
8. Garzon, R.; Calin, G.A.; Croce, C.M. MicroRNAs in Cancer. Annu. Rev. Med. 2009, 60, 167-179. [CrossRef] [PubMed]

9. Boeri, M.; Pastorino, U.; Sozzi, G. Role of microRNAs in lung cancer: microRNA signatures in cancer prognosis. Cancer J. 2012, 18, 268-274. [CrossRef] [PubMed]

10. Bianchi, F.; Nicassio, F.; Marzi, M.; Belloni, E.; Dall'olio, V.; Bernard, L.; Pelosi, G.; Maisonneuve, P.; Veronesi, G.; di Fiore, P.P. A serum circulating miRNA diagnostic test to identify asymptomatic high-risk individuals with early stage lung cancer. EMBO Mol. Med. 2011, 3, 495-503. [CrossRef] [PubMed]

11. Cortez, M.A.; Bueso-Ramos, C.; Ferdin, J.; Lopez-Berestein, G.; Sood, A.K.; Calin, G.A. MicroRNAs in body fluids-The mix of hormones and biomarkers. Nat. Rev. Clin. Oncol. 2011, 8, 467-477. [CrossRef] [PubMed]

12. Turchinovich, A.; Weiz, L.; Langheinz, A.; Burwinkel, B. Characterization of extracellular circulating microRNA. Nucleic Acids Res. 2011, 39, 7223-7233. [CrossRef] [PubMed]

13. Arroyo, J.D.; Chevillet, J.R.; Kroh, E.M.; Ruf, I.K.; Pritchard, C.C.; Gibson, D.F.; Mitchell, P.S.; Bennett, C.F.; Pogosova-Agadjanyan, E.L.; Stirewalt, D.L.; et al. Argonaute2 complexes carry a population of circulating microRNAs independent of vesicles in human plasma. Proc. Natl. Acad. Sci. USA 2011, 108, 5003-5008. [CrossRef] [PubMed]

14. Boeri, M.; Sestini, S.; Fortunato, O.; Verri, C.; Suatoni, P.; Pastorino, U.; Sozzi, G. Recent advances of microRNA-based molecular diagnostics to reduce false-positive lung cancer imaging. Expert Rev. Mol. Diagn. 2015, 15, 801-813. [CrossRef] [PubMed]

15. Sozzi, G.; Boeri, M.; Rossi, M.; Verri, C.; Suatoni, P.; Bravi, F.; Roz, L.; Conte, D.; Grassi, M.; Sverzellati, N.; et al. Clinical Utility of a Plasma-Based miRNA Signature Classifier Within Computed Tomography Lung Cancer Screening: A Correlative MILD Trial Study. J. Clin. Oncol. 2014, 32, 768-773. [CrossRef] [PubMed]

16. Boeri, M.; Verri, C.; Conte, D.; Roz, L.; Modena, P.; Facchinetti, F.; Calabro, E.; Croce, C.M.; Pastorino, U.; Sozzi, G. MicroRNA signatures in tissues and plasma predict development and prognosis of computed tomography detected lung cancer. Proc. Natl. Acad. Sci. USA 2011, 108, 3713-3718. [CrossRef] [PubMed]

17. Sestini, S.; Boeri, M.; Marchiano, A.; Pelosi, G.; Galeone, C.; Verri, C.; Suatoni, P.; Sverzellati, N.; La, V.C.; Sozzi, G.; et al. Circulating microRNA signature as liquid-biopsy to monitor lung cancer in low-dose computed tomography screening. Oncotarget 2015, 20, 32868-32877.

18. Fortunato, O.; Boeri, M.; Moro, M.; Verri, C.; Mensah, M.; Conte, D.; Caleca, L.; Roz, L.; Pastorino, U.; Sozzi, G. Mir-660 is downregulated in lung cancer patients and its replacement inhibits lung tumorigenesis by targeting MDM2-p53 interaction. Cell Death Dis. 2014, 5, e1564. [CrossRef] [PubMed]

19. Pastorino, U.; Bellomi, M.; Landoni, C.; de Fiori, E.; Arnaldi, P.; Picchio, M.; Pelosi, G.; Boyle, P.; Fazio, F. Early lung-cancer detection with spiral CT and positron emission tomography in heavy smokers: 2-year results. Lancet 2003, 362, 593-597. [CrossRef]

20. Fortunato, O.; Boeri, M.; Verri, C.; Conte, D.; Mensah, M.; Suatoni, P.; Pastorino, U.; Sozzi, G. Assessment of circulating microRNAs in plasma of lung cancer patients. Molecules 2014, 19, 3038-3054. [CrossRef] [PubMed]

21. Zhao, Y.; Simon, R. BRB-ArrayTools Data Archive for human cancer gene expression: A unique and efficient data sharing resource. Cancer Inform. 2008, 6, 9-15. [PubMed]

22. Dinse, G.E.; Lagakos, S.W. Nonparametric estimation of lifetime and disease onset distributions from incomplete observations. Biometrics 1982, 38, 921-932.T [CrossRef] [PubMed]

23. Vlachos, I.S.; Kostoulas, N.; Vergoulis, T.; Georgakilas, G.; Reczko, M.; Maragkakis, M.; Paraskevopoulou, M.D.; Prionidis, K.; Dalamagas, T.; Hatzigeorgiou, A.G. DIANA miRPath v.2.0: Investigating the combinatorial effect of microRNAs in pathways. Nucleic Acids Res. 2012, 40, W498-W504. [CrossRef] [PubMed]

24. Dweep, H.; Gretz, N. miRWalk2.0: A comprehensive atlas of microRNA-target interactions. Nat. Methods 2015, 12, 697. [CrossRef] [PubMed]

25. Li, M.; Zhang, S.; Wu, N.; Wu, L.; Wang, C.; Lin, Y. Overexpression of miR-499-5p inhibits non-small cell lung cancer proliferation and metastasis by targeting VAV3. Sci. Rep. 2016, 6, 23100. [CrossRef] [PubMed]

26. Sun, C.C.; Li, S.J.; Zhang, F.; Pan, J.Y.; Wang, L.; Yang, C.L.; Xi, Y.Y.; Li, D.J. Hsa-miR-329 exerts tumor suppressor function through down-regulation of MET in non-small cell lung cancer. Oncotarget 2016, 16, 21510-21527.

27. Zhang, S.; Shan, C.; Kong, G.; Du, Y.; Ye, L.; Zhang, X. MicroRNA-520e suppresses growth of hepatoma cells by targeting the NF-kB-inducing kinase (NIK). Oncogene 2012, 31, 3607-3620. [CrossRef] [PubMed] 
28. Li, H.; Liu, Y.W.; Wang, H.; Zhou, Q.; Li, J.-J.; Huang, A.; Qi, S.T.; Lu, Y.-T. MiR-519a functions as a tumor suppressor in glioma by targeting the oncogenic STAT3 pathway. J. Neurooncol. 2016, 128. [CrossRef] [PubMed]

29. Gao, Z.; Zhu, X.; Dou, Y. The miR-302/367 cluster: A comprehensive update on its evolution and functions. Open Biol. 2015, 5, 150138. [CrossRef] [PubMed]

30. Subramanyam, D.; Lamouille, S.; Judson, R.L.; Liu, J.Y.; Bucay, N.; Derynck, R.; Blelloch, R. Multiple targets of miR-302 and miR-372 promote reprogramming of human fibroblasts to induced pluripotent stem cells. Nat. Biotechnol. 2011, 29, 443-448. [CrossRef] [PubMed]

(C) 2016 by the authors; licensee MDPI, Basel, Switzerland. This article is an open access article distributed under the terms and conditions of the Creative Commons Attribution (CC-BY) license (http://creativecommons.org/licenses/by/4.0/). 